\title{
Coping materno da Síndrome de Down: identificando estressores e estratégias de enfrentamento
}

\author{
Maternal coping with Down Syndrome: Identifying \\ stressors and coping strategies
}

\author{
Luciana Bicalho REIS' \\ ORCID ID 0000-0001-6173-7514 \\ Kely Maria Pereira de PAULA ${ }^{2}$ \\ ORCID iD 0000-0001-9095-6556
}

\begin{abstract}
Resumo
O nascimento de uma criança com Síndrome de Down pode representar risco de estresse em mães, demandando estratégias de enfrentamento. Este estudo identificou os principais estressores e as estratégias de enfrentamento adotadas por nove mães de crianças com essa patologia. As participantes responderam a um roteiro de entrevista adaptado da Motivational Theory of Coping Scale - 12. Os principais estressores identificados foram: preconceito social, rotina de atendimentos, problemas de comportamento da criança, alterações do desenvolvimento e problemas de saúde. A maioria adotou estratégias pertencentes às categorias adaptativas: Autoconfiança, Busca de Suporte, Busca de Informação, Resolução de Problemas, Acomodação e Negociação. Estratégias de enfrentamento de categorias mal adaptativas foram empregadas por algumas mães: Fuga, Isolamento, Submissão e Desamparo. O emprego de estratégias adaptativas aumenta as chances de resultado positivo para saúde mental materna a médio e longo prazos.
\end{abstract}

Palavras-chave: Coping; Estresse; Mães; Síndrome de Down.

\begin{abstract}
The birth of a child with Down Syndrome may represent a risk of maternal stress, thus requiring the use of coping strategies. This research identified the main stressors and coping strategies adopted by nine mothers of children with Down Syndrome. The participants answered the questions of an interview script adapted from the Motivational Theory of Coping Scale - 12. The main stressors identified were as follows: social prejudice, routine care, child behavior problems, developmental disorders, and health issues. Most mothers adopted adaptive strategies such as Self-reliance, Support Seeking, Information Seeking, Problem Solving, Negotiation, and Accommodation. Some mothers employed maladaptive coping strategies such as Escape, Social Isolation, Submission, and Helplessness. The use of adaptive strategies increases the chances of medium- and long-term benefits regarding maternal mental health.
\end{abstract}

Keywords: Coping; Mothers; Down Syndrome; Stress.

$\checkmark v \nabla v$

1 Universidade Federal do Espírito Santo, Centro de Ciências Humanas e Naturais, Departamento de Psicologia. Av. Fernando Ferrari, 514 , Goiabeiras, 29075-910, Vitória, ES, Brasil. Correspondência para/Correspondence to: L.B. REIS. E-mail: <lucianabreis@hotmail.com>

2 Universidade Federal do Espírito Santo, Programa de Pós-Graduação em Psicologia, Departamento de Psicologia Social e do Desenvolvimento. Vitória, ES, Brasil.

Artigo elaborado a partir da tese de L.B. Reis, intitulada "Proposta de intervenção da mediação de mães de crianças com Síndrome de Down". Universidade Federal do Espírito Santo, 2015.

$\boldsymbol{\nabla} \mathbf{v}$

Como citar este artigo/How to cite this article

Reis, L. B., \& Paula K. M. P. (2018). Coping materno da Síndrome de Down: identificando estressores e estratégias de enfrentamento. Estudos de Psicologia (Campinas), 35(1), 77-88. https://doi.org/10.1590/1982-02752018000100008 
Ter um filho com deficiência não é, necessariamente, uma situação problema para a família, embora represente risco para futuras dificuldades (Barbosa, Balieiro, \& Pettengill, 2012; Fiamenghi Jr. \& Messa, 2007; Rooke \& Pereira-Silva, 2016). Diversos autores têm identificado altos níveis de estresse em famílias de crianças com deficiências ou atrasos no desenvolvimento (Al-Qaisy, 2012; Karasavvidis et al., 2011; Ribeiro, Porto, \& Vandenberghe, 2013), mas a mãe é normalmente o membro mais afetado por ser a cuidadora direta (Albuquerque, Pereira, Fonseca, \& Canavarro, 2012; Cherubini, Bosa, \& Bandeira, 2008; Silva \& Dessen, 2003). Quanto maior for o comprometimento e a dependência da criança, maiores serão as sobrecargas físicas, psicológicas, sociais e financeiras da família e dos genitores individualmente, ampliando, portanto, o nível de estresse. Nesses casos, estariam também associados os sentimentos de ansiedade e preocupação quanto às possibilidades de sobrevivência e desenvolvimento da criança, além da necessidade de cuidados prolongados (Silva \& Dessen, 2003).

Diferentes estudos identificaram correlação entre estresse parental e problemas de comportamento da criança (Al-Qaisy 2012; Karasavvidis et al., 2011), estresse e nível de apoio social recebido pela família (Hassall, Rose, \& McDonald, 2005), gastos financeiros em função da deficiência da criança e dificuldade dos pais em ter vida social ativa (Al-Qaisy, 2012) e, ainda, problemas no relacionamento conjugal (Karasavvidis et al., 2011). Gohel, Mukherjee e Choudhary (2011) apontam que, quanto maior o nível de dependência da criança para as atividades da vida diária, maior o impacto da deficiência sobre a família. Segundo os autores, o apoio social, em especial de outras famílias de crianças com deficiência, foi capaz de diminuir o nível de estresse entre os participantes de seu estudo.

Desde a década de 1970, teorias psicológicas têm apontado para a necessidade de se considerar a avaliação cognitiva e as respostas de enfrentamento (coping) como variáveis integrantes do fenômeno de estresse (Seidl, Tróccoli, \& Zannon, 2001). Dessa forma, o estresse passa a ser estudado por vários autores a partir de um modelo interativo (Transactional Modelo of Stress and Coping), que compreende estresse e enfrentamento a partir da interação entre organismo e seu ambiente e não como propriedade de um ou de outro (Lazarus, 1993; Skinner \& Zimmer-Gembeck, 2009). Dessa interação resultam a saúde mental ou psicopatologias (Zimmer-Gembeck \& Skinner, 2016).

Segundo Skinner, Edge, Altman e Sherwood (2003), o enfrentamento é um construto usado para agrupar e organizar um conjunto de ações adotadas pelos indivíduos para lidar com experiências estressantes, envolvendo comportamentos, percepções e cognições. Logo, não deve ser analisado como crença ou um comportamento específico que pode ser identificado, mas como um processo resultante da interação entre o organismo e o estímulo considerado estressor, o que torna sua avaliação complexa (Grassi-Oliveira, Daruy Filho, \& Brietzke, 2008). Neste sentido, Skinner e Welborn (1994, p.111, tradução nossa) afirmam que o coping ou enfrentamento é um "construto organizacional que descreve como as pessoas regulam seu comportamento, emoção e motivação sob condições psicofisiológicas de estresse ... e de que maneira se esforçam para manter, restaurar, renovar e reparar a satisfação de suas necessidades". Refere-se, portanto, a um conjunto de recursos cognitivos e comportamentais apresentados diante de demandas e exigências internas e externas consideradas estressoras pelo indivíduo (Seidl et al., 2001; Skinner et al., 2003).

Skinner e Wellborn (1994) e Skinner et al. (2003) propõem a Teoria Motivacional do Coping (TMC) em que o enfrentamento é entendido a partir de um sistema hierárquico de Categorias. No nível mais elementar estão as respostas (cognitivas, comportamentais ou emocionais) dos indivíduos para lidar com situações estressantes, o que Skinner denominou instâncias ou comportamentos de coping (instances) (Skinner \& Zimmer-Gembeck, 2009, Zimmer-Gembeck \& Skinner, 2011) e incluem o que a pessoa faz ou pensa. No nível superior imediato se localizam as Estratégias de Enfrentamento (Ways of Coping), que seriam subcategorias/ agrupamentos de ações frente ao estresse. As Estratégias de Enfrentamento são classificadas em 
termos de seu núcleo e de sua função compondo 12 Categorias de coping de alta ordem (coping families) organizadas de acordo com sua função no processo adaptativo (Skinner et al., 2003; Skinner \& Zimmer-Gembeck, 2009).

Na perspectiva da TMC, há seis Categorias de alta ordem com possível desfecho positivo no processo adaptativo, sendo elas Resolução de Problemas, Busca de Informação, Negociação, Acomodação, Busca de Suporte e Autoconfiança; e seis Categorias mal adaptativas, com maior probabilidade de prejuízos ou resultados negativos no desenvolvimento, identificadas como Desamparo, Fuga, Oposição, Submissão, Delegação e Isolamento Social (Zimmer-Gembeck \& Skinner, 2011). Embora não se possa classificar a priori o que seriam estratégias adaptativas ou mal adaptativas, algumas Estratégias de Enfrentamento têm maior possibilidade de fazer com que o indivíduo, a longo prazo, consiga lidar melhor com a situação de estresse e tenha resultados mais satisfatórios no desenvolvimento (Skinner et al., 2003; Ramos, Enumo, \& Paula, 2015).

No Brasil, estudos têm procurado investigar o enfrentamento na perspectiva da TMC com diferentes populações, como o enfrentamento de mães diante da cardiopatia congênita de seus bebês (Gonçalves, Reis, \& Vicente, 2015); de profissionais de saúde diante da morte fetal (Cravinho \& Cunha, 2015); do tratamento de câncer em crianças (Motta et al., 2015; Hostert, Motta, \& Enumo, 2015; Motta \& Enumo, 2010). Nessa perspectiva, para a compreensão das Estratégias de Enfrentamento, também é preciso considerar a natureza do estressor com o qual se está lidando, já que pode ter para o indivíduo um caráter de desafio ou ameaça às necessidades psicológicas: competência, autonomia e relacionamento (Skinner, 1999; 2007). A necessidade de competência envolve a interação positiva com o ambiente em busca de resultados positivos, alcançando o que se deseja; já a necessidade de autonomia envolve a possibilidade de o indivíduo ser capaz de fazer escolhas; e a de relacionamento caracteriza-se pela necessidade primordial de manter relacionamento próximo com o outro (Skinner \& Wellborn, 1994).
Deste modo, as Estratégias de Enfrentamento são adaptativas na medida em que permitem ao sujeito regular seus afetos e comportamentos, atendendo a uma dessas três necessidades básicas. Assim, com base na hipótese de que a deficiência da criança, ou condições dela decorrentes, pode ser fonte de estresse para pais, este trabalho procurou identificar os estressores e analisar as estratégias de enfrentamento empregadas por mães de crianças com Síndrome de Down.

\section{Método}

\section{Participantes}

Participaram da pesquisa nove mães de crianças com Síndrome de Down e idade entre 2 e 5 anos, atendidas em instituições de reabilitação da Grande Vitória (ES), compondo uma amostra de conveniência. O critério de inclusão foi o de ter um filho com Síndrome de Down e os de exclusão, a presença de outras alterações no desenvolvimento associadas à Síndrome, como, por exemplo, autismo e paralisia cerebral ou história pregressa materna de transtorno mental verificada por meio de informação dada pela própria mãe.

A entrevista foi realizada na residência das participantes ou em uma sala reservada nas instituições em que as crianças eram atendidas, garantindo condições adequadas de bem-estar e sigilo dos dados conforme Resolução n 466/12 do Conselho Nacional de Saúde (2012). O estudo foi aprovado pelo Comitê de Ética em Pesquisa da Universidade Federal do Espírito Santo, com Parecer no 198.850 (aprovado em 07/01/2013), tendo todas as mães assinado o Termo de Consentimento Livre e Esclarecido. As entrevistas foram gravadas em áudio para posterior transcrição.

\section{Instrumentos}

Para coleta de dados, as mães responderam à Entrevista de Avaliação do Enfrentamento - roteiro de livre tradução e adaptação da Motivational Theory of Coping Scale-12 (MTC-12) de Lees 
(2007), baseada na TMC (Skinner et al., 2003; Skinner \& Wellborn, 1994), empregada, em versões adaptadas, em estudos no país (Cravinho \& Cunha, 2015). A entrevista caracteriza-se por um roteiro semiestruturado com 12 perguntas principais relacionadas às categorias de enfrentamento da TMC: (1) Autoconfiança; (2) Busca por Suporte; (3) Resolução de Problemas; (4) Busca de Informação; (5) Acomodação; (6) Negociação; (7) Delegação; (8) Isolamento; (9) Desamparo; (10) Fuga; (11) Submissão; e (12) Oposição. A cada pergunta, a mãe deveria responder "sim" ou "não" para o emprego de Estratégias de Enfrentamento referentes à categoria de alta ordem, justificando a resposta afirmativa a partir das perguntas "como" e "como se sentia". Tendo em vista que alguns anos já se passaram desde o nascimento e diagnóstico do filho, foi acrescentada à entrevista original a seguinte pergunta: "Considerando tudo que você passou desde o nascimento de seu filho, há algo que considera atualmente como sendo mais difícil na situação de ter um filho com Síndrome de Down?".

A análise dos dados foi do tipo quantiqualitativa. As entrevistas foram transcritas e analisadas à luz da TMC (Skinner et al., 2003). Por meio dos relatos verbais, foi possível verificar o que a mãe fazia, pensava ou sentia diante do estressor, efetuando-se a identificação das Estratégias de Enfrentamento e, por conseguinte, das categorias de alta ordem (coping families). Os dados também foram submetidos à estatística descritiva para compreensão da predominância das Estratégias de Enfrentamento levantadas nesses relatos.

\section{Resultados e Discussão} a M9) tinham idades entre 32 e 51 anos, sendo a média 42. O nível de escolaridade variou do iletramento à pós-graduação. As crianças tinham idades entre 2 e 5 anos (designadas C1 a C9, conforme suas mães), com média de 3,9, e a maioria era de meninos $(n=5)$. O tempo decorrente desde o diagnóstico era exatamente a idade da criança, já que todas foram diagnosticadas ao nascimento ( $\mathrm{C} 1-3$ anos, C2 -5 anos, C3 -3 anos, C4 -3 anos, C $5-4$ anos, C $6-3$ anos, C $7-5$ anos, (8 - 2 anos, (9 - 3 anos).

\section{Identificando fontes de estresse do cuidador}

A partir dos relatos verbais das mães, cinco estressores foram identificados, sendo eles Preconceito Social sofrido pela criança $(n=3)$, Rotina de Atendimentos $(n=2)$, Desenvolvimento $(n=2)$, Problemas de Comportamento $(n=2)$ e Problemas de saúde da criança $(n=1)$. Uma das mães indicou mais de uma fonte de estresse (Preconceito Social e Desenvolvimento da Criança).

O Preconceito Social foi o estressor mais indicado pelas mães $(n=3)$, estando relacionado a situações em que pessoas comentam ou perguntam de modo desrespeitoso sobre a condição da criança ou ao temor de que o filho seja excluído em alguma situação de interação social (M1, M2 e M6). Em seguida, em termos de número de respostas, tem-se Rotina de Atendimentos (M4, M5) ( $n=2)$ e Problemas de Comportamento da Criança (M7, M9) $(n=2)$. Algumas mães relataram que todos os cuidados com o filho desde o nascimento (internação, exames médicos, cirurgias e terapias) acabam gerando intenso desgaste físico (cansaço) e mental (preocupação, hipervigilância, temor).

Para duas participantes (M7, M9), as fontes de estresse advêm das dificuldades de comportamento da criança $(n=2)$, descritas como voluntariosas e hiperativas. Duas mães (M1, M3) relataram a preocupação de que as crianças se desenvolvam bem, tornem-se independentes e consigam se alfabetizar. Outra mãe (M8) refere-se aos problemas de saúde $(n=1)$ decorrentes da baixa imunidade do filho como sendo uma fonte de preocupação constante. Os achados corroboram dados de outros estudos que identificaram estressores semelhantes, como problemas de comportamento da criança (Al-Qaisy, 2012; Hassall et al., 2005; Karasavvidis et al., 2011; Ribeiro et al., 2013; Weiss, Sullivan, \& Diamond, 2003); preconceito e isolamento social (Al-Qaisy, 2012); desenvolvimento da criança (Gohel et al., 2011; Silva \& Dessen, 2004); e problemas de saúde (Heiman, 2002). 


\section{Categorias de enfrentamento empregadas pelas mães}

A partir da análise das entrevistas, considerando o que as participantes relataram fazer, pensar ou sentir diante dos estressores identificados, o seguinte repertório de enfrentamento foi levantado: Autoconfiança, apresentada pela maioria $(n=8)$, seguida de Busca de Suporte, Resolução de Problemas e Busca de Informação $(n=7)$, Acomodação $(n=6)$, Fuga $(n=3)$, Isolamento e Submissão $(n=2)$, além de Negociação e Desamparo $(n=1)$, que foram as Categorias menos relatadas. Nenhuma participante relatou Estratégias de Enfrentamento pertencentes às Categorias Delegação e Oposição.

Todas as mães adotaram estratégias pertencentes às Categorias de enfrentamento adaptativas, sendo que algumas relataram exclusivamente esse tipo de estratégia (M1, M3, M4, M6 e M8). Outras, embora com menor frequência, também adotaram estratégias que integram as Categorias mal adaptativas (M7 e M9). Uma mãe demonstrou equivalência no emprego de estratégias de ambos os grupos (M2) e somente uma participante (M5) relatou mais estratégias de Categorias mal adaptativas que positivas frente aos estressores da condição da criança ou do contexto. Esses dados podem ser visualizados na Figura 1.

A entrevista também permitiu identificar as Estratégias de Enfrentamento e os comportamentos

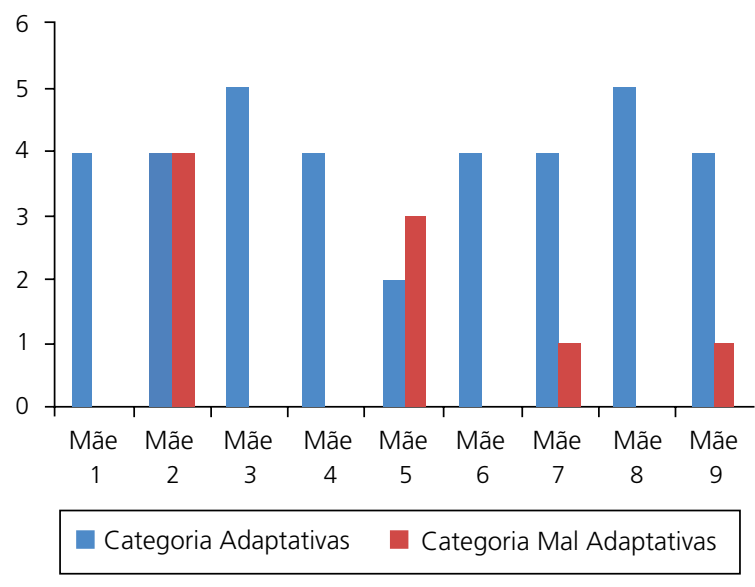

Figura 1. Distribuição das categorias de alta ordem $(N=9)$. de coping empregados pelas mães ao serem indagadas sobre o que efetivamente faziam e como se sentiam no contexto de estresse. Ao todo, 20 Estratégias de Enfrentamento foram apresentadas pelas participantes, totalizando 52 citações, sendo que, destas, $82,70 \%$ pertenciam às Categorias adaptativas: Busca de Informação $(21,15 \%)$, seguida por Busca de Suporte (19,23\%), Autoconfiança, Resolução de Problemas e Acomodação (cada uma representando $13,46 \%$ das citações) e Negociação (1,92\%) (Tabela 1).

Estratégias da Categoria Busca de Informação foram relatadas por sete das nove mães, como exemplificado no relato de M1: "Quando eu tenho tempo eu sento lá na frente do computador, eu leio artigos [...] . Quando ele fez 1 ano [...]. Aí eu comecei a pesquisar [...]" (Estressor Desenvolvimento da Criança; Estratégias de Enfrentamento Ler; Busca de Informação). Nesse caso, como o estressor é avaliado como desafio, a meta é encontrar contingências adicionais que ajudem a lidar com a situação (Ramos et al., 2015). Nas entrevistas, as mães apresentaram diferentes comportamentos visando se informar sobre o estressor, como ler arti-gos, assistir a programas de televisão que discutissem sobre a deficiência e perguntar aos médicos sobre a condição da criança.

Ao usar várias estratégias para conhecer a Síndrome de Down, as mães sentiram-se mais competentes para lidar com dificuldades relacionadas à deficiência. A Busca de Informação é sobremodo importante, tanto na ocasião do nascimento quanto ao longo do desenvolvimento do filho, favorecendo o enfrentamento das dificuldades e o ajustamento de expectativas por parte dos pais. Além disso, adquirir informações parece ajudá-los a ter maior sentimento de controle sobre a situação, podendo diminuir a ansiedade.

Ao adotar estratégias da categoria Busca de Suporte, a pessoa também avalia o estressor como um desafio a ser superado e sua ação é direcionada ao ambiente, buscando encontrar nele meios de atender à necessidade psicológica de Relacionamento. As Estratégias de Enfrentamento apresentadas pelas mães, que somam 19,23\% das citações, foram Busca de Conforto, Busca 
Repertório de coping da Síndrome de Down no grupo de mães ( $N=9$ )

\begin{tabular}{|c|c|c|c|c|}
\hline \multirow{14}{*}{ 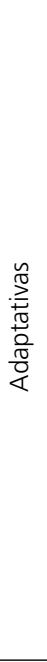 } & Categorias de Enfrentamento (coping families) & EE/comportamentos de coping & $f$ & Total por categoria (\%) \\
\hline & \multirow{2}{*}{ Autoconfiança } & Regulação Emocional & 4 & \multirow{2}{*}{13,46} \\
\hline & & Regulação Comportamental & 3 & \\
\hline & \multirow{3}{*}{ Busca de Suporte } & Busca de Conforto & 4 & \multirow{3}{*}{19,23} \\
\hline & & Busca de Contato & 2 & \\
\hline & & Ajuda Instrumental & 4 & \\
\hline & Resolução de Problemas & Ação Instrumental & 7 & 13,46 \\
\hline & \multirow{3}{*}{ Busca de Informação } & Ler & 7 & \multirow{3}{*}{21,15} \\
\hline & & Observar & 1 & \\
\hline & & Perguntar aos Outros & 3 & \\
\hline & \multirow{3}{*}{ Acomodação } & Reestruturação Cognitiva & 1 & \multirow{3}{*}{13,46} \\
\hline & & Aceitação & 4 & \\
\hline & & Minimização & 2 & \\
\hline & Negociação & Persuasão & 1 & 1,92 \\
\hline \multirow{7}{*}{ 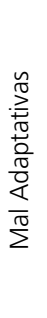 } & Isolamento & Afastamento Social & 1 & \multirow{2}{*}{3,84} \\
\hline & 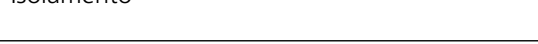 & Evitar outras Pessoas & 1 & \\
\hline & \multirow{2}{*}{ Desamparo } & Passividade & 1 & \multirow{2}{*}{3,84} \\
\hline & & Exaustão Cognitiva & 1 & \\
\hline & \multirow{2}{*}{ Fuga } & Pensamento Desejoso & 1 & \multirow{2}{*}{5,76} \\
\hline & & Evitação Comportamental & 2 & \\
\hline & Submissão & Ruminação & 2 & 3,84 \\
\hline
\end{tabular}

Nota: EE: Estratégias de Enfrentamento.

de Contato e Ajuda Instrumental (Skinner et al., 2003). As fontes de apoio mais citadas foram a família ( $n=7)$, outras famílias de crianças com deficiência $(n=3)$ e profissionais das instituições que frequentam $(n=3)$. O suporte social tem sido identificado como recurso para enfrentamento de condições adversas (Skinner \& Edge, 2002), tendo importante efeito sobre a saúde mental de pais de crianças com deficiência (Matsukura, Marturano, Oishi, \& Borasche, 2007) e de mães no período pós-parto (Razurel, Kaiser, Sellenet, \& Epiney, 2013). A ajuda instrumental também foi uma estratégia apresentada por algumas mães, as quais recorrem aos familiares e profissionais para lidar com o estressor. A participação de pessoas na construção de uma rede de apoio para as atividades cotidianas e nos cuidados com a própria criança pode amenizar os efeitos estressores na mãe.

Na categoria Autoconfiança, foram citadas 82 as Estratégias de Enfrentamento regulação emocional e regulação comportamental. A Autoconfiança envolve a necessidade psicológica de manter relacionamentos e é empregada quando o sujeito se sente desafiado e confiante, agindo de modo a proteger recursos sociais disponíveis, conforme relato de M4: "Mas eu consigo [...]. Eu sinto força. Não me sinto cansada" (Estressor Rotina de Tratamento da Criança; Estratégias de Enfrentamento Regulação Emocional; Autoconfiança).

Sentir-se confiante e capaz de lidar com um estressor é de fundamental importância para enfrentamento de situações adversas. Pais e mães que se consideram eficientes em seu papel ao lidar com o filho com deficiência (Ribeiro et al., 2013) e que se mostram positivos diante da situação apresentam menores níveis de estresse e melhor ajustamento a longo prazo (Trute, Benzies, \& Worthington, 2012). Assim, quando as mães relatam sentirem-se capazes de lidar com as dificuldades advindas da condição da criança, tanto emocionalmente quanto do ponto de vista comportamental, pode-se afirmar que 
apresentam autoconfiança, o que favorece o melhor ajustamento e enfrentamento da situação.

Na Resolução de Problemas, a Estratégia de Enfrentamento apresentada pelas mães foi a Ação Instrumental. Nessa categoria de alta ordem, as ações objetivam a resolução da situação, atendendo à necessidade de competência, como identificado no relato de M4: "[...] arrumo ela [...] levo pra onde tem que levar [...]. Procuro resolver. É o médico... qualquer coisa. Eu consigo, faço 'pra' ela" (Estressor Rotina de Tratamento da Criança; Estratégias de Enfrentamento Ação Instrumental; Resolução de Problemas).

Ao se depararem com as dificuldades que envolviam a deficiência do filho, as mães buscaram diferentes soluções, como, por exemplo, conseguir tratamento médico ou atendimentos para a criança ou reunir-se com a equipe pedagógica da escola para traçar estratégias de modificação de seu comportamento. O que há em comum nessas ações é a intenção de resolver ou ao menos lidar ativamente com o problema, o que aumenta o senso de competência, favorecendo a adoção de novas ações com este fim. As Estratégias de Enfrentamento pertencentes à Resolução de problemas indicam um enfrentamento ativo do estressor, procurando meios de lidar com ele.

A Acomodação, no processo adaptativo, relaciona-se à necessidade psicológica de autonomia. Estratégias como Reestruturação Cognitiva (mudar pensamentos, ideias e sentimentos acerca do estressor), Minimização (atribuir menor importância ao estressor) e Aceitação (ajustar-se de forma autônoma ao estressor) permitem um ajuste das preferências às opções disponíveis de forma flexível, como destacado no relato de M9: "Eu aceito, porque o L. é como eu te falei, é da personalidade dele, né? Que é muito forte" (Estressor Problemas de Comportamento Criança; Estratégias de Enfrentamento Aceitação; Acomodação). Nesse sentido, estressores também são percebidos como desafio e não como ameaça (Skinner et al., 2003). Assim, algumas mães, de diferentes maneiras, indicaram ter obtido êxito para se ajustar/adaptar à situação adversa, reestruturando determinados pensamentos relativos à criança, seja pelas estratégias aceitação seja através da minimização da Síndrome de Down, como no caso da avaliação da teimosia da criança como uma característica de sua personalidade.

Ainda dentre as categorias Adaptativas, somente uma mãe (M8) indicou adotar a Estratégia de Enfrentamento Persuasão, pertencente à Negociação. Por meio dela, a pessoa procura encontrar novas opções, dirigindo sua ação ao contexto a fim de alcançar ou manter sua autonomia. Um exemplo disso é o relato de uma mãe que precisou persuadir o marido a aceitar algum tratamento novo para a criança.

Além das Estratégias de Enfrentamento adaptativas até aqui apresentadas, também foram identificadas categorias Mal Adaptativas: Fuga, Isolamento, Submissão e Desamparo. Nesse caso, o estressor, ao invés de ser considerado um desafio, é percebido como ameaça a uma das três necessidades psicológicas (Relacionamento, Autonomia e Competência) (Skinner et al., 2003).

Embora de modos distintos, duas mães (M2 e M5) também relataram sensação de falta de competência, o que restringia as ações para lidar com os estressores da deficiência. Três participantes (M2, M5, M9) apresentaram Estratégias de Enfrentamento que remetem à Fuga. Quando da percepção de ambientes não contingentes associados à impossibilidade de manejo das adversidades, as mães apresentaram as Estratégias de Enfrentamento Pensamento Desejoso $(n=2)$, que consiste na vontade de fugir do estressor, e Evitação Comportamental $(n=1)$, traduzida em comportamentos no sentido de se afastar de determinada situação. A consciência do pensamento de fugir da situação estressora parece gerar, para uma das mães (M5), emoções negativas como a culpa, por associá-la à vontade de fugir da própria criança, como destacado no relato: " $A i$, meu Deus do céu, é pecado né? Querer fugir, todo mundo pensa, né? Mas depois a gente volta atrás. Filho é maior do que qualquer coisa. Sempre que me vem esse pensamento, eu peço logo perdão a Deus" (Estressor Rotina de Atendimentos da Criança; Estratégias de Enfrentamento Pensamento Desejoso; Fuga). 
Ao longo da entrevista, M2 relatou tristeza, incômodo e raiva por vivenciar preconceito dirigido à criança em inúmeras situações sociais (interações no transporte coletivo, vizinhança, entre outros): "[...] eu pego e fujo [...] tem hora que não dá [referindo--se ao fato de preferir sair do lugar]" (Estressor Preconceito Social; Estratégias de Enfrentamento Evitação Comportamental; Fuga). Acredita-se que a Estratégia de Enfrentamento de Fuga (evitar am-bientes e pessoas preconceituosos) permita à $\mathrm{M} 2$ diminuir o estresse decorrente da situação de forma imediata. No entanto, é possível supor que a médio e longo prazos essa estratégia, se empregada com muita frequência, poderá mostra-se mal adaptativa por trazer riscos de restrições de socialização à criança e à família e, ainda, porque o frequente emprego de Estratégias de Enfrentamento mal adaptativas está associado a condições psíquicas como ansiedade e depressão (Zimmer-Gembeck \& Skinner, 2015, 2016).

Afastamento social foi uma Estratégia de Enfrentamento identificada somente no relato de duas mães, sendo relacionada ao Isolamento, que consiste em adotar comportamentos visando se distanciar de contextos sociais não apoiadores, preferindo manter-se isolada (Skinner et al., 2003). Para M2: "[...] tem hora que eu não tô pra ninguém, nem pra vizinha. Tem hora que eu me isolo. [...] Aí, eu fico sozinha pensando, fico pensando nas coisas. [...] eu choro pra minha raiva sair um pouco" (Estressor Preconceito Social; Estratégias de Enfrentamento Afastamento Social; Isolamento). Como se percebe no relato, o emprego da Estratégia de Enfrentamento mal adaptativa aparece acompanhado de sentimentos negativos, como a raiva, o que corrobora achados da literatura (Zimmer-Gembeck \& Skinner, 2015, 2016).

Entre as categorias de alta ordem mal adaptativas apresentadas pelas mães $(n=2)$, a Submissão foi relatada em algumas situações onde havia percepção de ameaça à necessidade de autonomia, com comportamentos característicos da Estratégia de Enfrentamento Ruminação. Esse é um tipo de pensamento denominado intrusivo, podendo gerar emoções negativas e problemas físicos (Zanon, Borsa, Bandeira, \& Hutz, 2012), exemplificados nos relatos de M7 e M2,

84 escola. Às vezes eu perco o sono de tanto pensar nisso" (Estressor Problemas de Comportamento da criança; Estratégias de Enfrentamento Ruminação; Submissão); "É, eu fico assim, tem hora que eu fico magoada [...]. Imagine quando ele tiver entendendo, [...] imagina ele grande. Eu fico pensando essas coisas. [...] Ah, eu fico muito triste mesmo, muito magoada" (Estressor Preconceito Social; Estratégias de Enfrentamento Ruminação; Submissão). A presença de pensamentos intrusivos com caráter de ruminação tem sido associada a condições de ansiedade, depressão, desajustamento psicossocial e vulnerabilidade (Zanon et al., 2012).

Por fim, entre as mal adaptativas, ainda foram identificadas Estratégias de Enfrentamento da categoria Desamparo, em que o estressor é avaliado como uma ameaça à necessidade da pessoa de se sentir competente, levando-a a perceber limites para as ações. Passividade e Exaustão cognitiva foram duas Estratégias de Enfrentamento identificadas nessa categoria. A Passividade foi identificada quando a mãe se percebia limitada em sua capacidade de enfrentar as adversidades, mantendo-se inativa, como no relato de M2: "Porque não adianta, né? Falar, expressar nada, tem que deixar pra lá. [...] Deixa o pensamento deles pra lá. Eu no meu, eles no deles" (Estressor Preconceito Social; Estratégias de Enfrentamento Passividade; Desamparo). Já na Exaustão Cognitiva, há uma sensação de cansaço e de incapacidade para lidar com as dificuldades, como se faltassem forças e recursos, como aponta M5: "Às vezes eu tomo remédio. Tem semana que eu tô [...] muito cansada, tô muito ansiosa. Aí eu tenho que fazer o uso de um remédio [...] , é muita coisa. Realmente se você for encarar tudo, pra você encarar tudo isso, assim, de cara limpa... tem que ser muito zen" (Estressor Rotina de Atendimento; Estratégias de Enfrentamento Exaustão Cognitiva; Desamparo). Como se percebe nos relatos e diante de estressores diferentes, as duas participantes sentem que não têm recursos para lidar com a situação, experimentando sensação de desamparo.

A partir da análise dos dados, pode-se afirmar, em consonância com alguns autores (Fiamenghi Jr. \& Messa, 2007), que o nascimento de uma criança com deficiência representa situação de risco para dificuldades afetivas dos pais. A maneira como a 
família lida com esse acontecimento depende do contexto cultural em que se encontra, da natureza da deficiência, das características de personalidade dos pais (Lidz, 2003), do tempo decorrido desde o diagnóstico e do suporte social e emocional recebido (Rooke \& Pereira-Silva, 2016; Taanila, Syrjälä, Kokkonen, \& Järvelin, 2002).

Nota-se que a maioria das mães (M1, M3, M4, M6 e M8) relatou, diante dos estressores, a presença de Estratégias de Enfrentamento adaptativas (82,70\%), distribuídas em Busca de Informação (21,15\%), Busca de Suporte (19,23\%), Autoconfiança (13,46\%), Resolução de Problemas $(13,46 \%)$, Acomodação (13,46\%) e Negociação $(1,92 \%)$, mas também apresentaram Estratégias de Enfrentamento consideradas mal adaptativas, embora em menor frequência (M7 e M9). Somente uma participante (M2) demonstrou equivalência no emprego de estratégias de ambas as categorias de alta ordem e apenas M5 relatou apresentar mais ocorrência de estratégias de categorias mal adaptativas frente ao estressor Rotina de Atendimentos da Criança. Além disso, os dados indicam que algumas mães (M2 e M5) apresentam necessidade de receber assistência psicológica por fazerem uso de Estratégias de Enfrentamento de categorias mal adaptativas com mais frequência, havendo, inclusive, o relato de sentimento de mal-estar psíquico. Nessa condição, o risco para ansiedade e depressão é maior, podendo comprometer o vínculo emocional com a criança (Piccinini, Frizzo, Brys, \& Lopes, 2011).

\section{Considerações Finais}

Tendo decorrido alguns anos após o nascimento da criança, é possível à mãe superar as dificuldades iniciais referentes à deficiência, como identificado em outros estudos (Heiman, 2002; Rooke \& Pereira-Silva, 2016). Entretanto, novas condições relacionadas ao diagnóstico se apresentam no curso de desenvolvimento da criança, sendo percebidas como estressores. Dessa maneira, é necessário à mãe um enfrentamento adequado, visando o reestabelecimento do equilíbrio emocional.

Na análise do construto Enfrentamento, em termos metodológicos, o formato de entrevista adotado, baseada na MTC-12 (Lees, 2007), se mostrou adequado à identificação das Categorias de alta ordem, das Estratégias de Enfrentamento e comportamentos de coping mais frequentemente relatados pelas mães diante de estressores cotidianos, viabilizando uma análise da funcionalidade das estratégias adotadas (Skinner, 1999; 2007; Skinner et al., 2003). O acréscimo da pergunta inicial à entrevista permitiu a identificação de estressores atuais e das formas de enfrentamento empregadas. Isso é adequado ao estudo do coping, o qual, sempre que possível, deve ser temporalmente próximo ao processo e não rememorativo (Lees, 2007).

Considera-se que o emprego predominante de Estratégias de Enfrentamento adaptativas é favorável ao bem-estar da mãe e ao desenvolvimento da criança. Aceitar a condição da criança, buscar informações, ter sentimentos e atitudes positivas diante das dificuldades advindas da deficiência e a busca de suporte social constituem-se formas de enfrentamento mais adaptativas à deficiência (Taanila et al., 2002). Dessa maneira, é possível afirmar que as chances de desfechos positivos relativos à saúde mental da mãe, bem como para o desenvolvimento da criança serão maiores.

É relevante conhecer as Estratégias de Enfrentamento apresentadas por mães ao lidar com os estressores advindos da deficiência dos filhos para a elaboração e aplicação de programas de intervenção psicológica em instituições de saúde, instrumentalizando melhor a população-alvo na adoção de ações de manejo e controle adequados do estresse. Deve-se oferecer aos pais apoio emocional na ocasião do diagnóstico, com possibilidade de obter informações contínuas ao longo do desenvolvimento, tendo em vista os novos desafios que cada fase apresenta, além de garantir a oportunidade de trocar experiências em grupos de pais. Portanto, a oferta de serviços de apoio à família (Summers, Behr, \& Turnbull,1988) tem o potencial de ampliar recursos adaptativos de enfrentamento da deficiência. Certamente é um desafio para os profissionais de saúde propor mudanças no padrão de enfrentamento materno mal adaptativo diante de estressores que não 
podem ser alterados, como ocorre no diagnóstico de deficiências, as quais geram problemas de saúde e limitações no desenvolvimento infantil. Porém, mediante intervenções adequadas e contínuas, amplia-se a chance de bem-estar materno, melhorando a qualidade de vida da família.

\section{Colaboradores}

L.B. REIS contribuiu na concepção e desenho do artigo, análise e interpretação dos dados, na discussão dos resultados e na elaboração do artigo. K.M.P. PAULA contribuiu na análise e interpretação dos dados e na discussão dos resultados, e revisão e aprovação da versão final do artigo.

\section{Referências}

Albuquerque, S., Pereira, M., Fonseca, A., \& Canavarro, M. C. (2012). Impacto familiar e ajustamento de pais de crianças com diagnóstico de anomalia congênita: influência dos determinantes da criança. Revista de Psiquiatria Clínica, 39(4), 136-41. https://doi. org/10.1590/S0101-60832012000400004

Al-Qaisy, L. M. (2012). Mothers' stress in families of children with mental handicap. Asian Social Science, 8(2). https://doi.org/10.5539/ass.v8n2p80

Barbosa, M. A. M., Balieiro, M. M. F. G., \& Pettengill, M. A. M. (2012). Family-centered care in the context of children with disabilities and their families: A reflective review. Texto and Contexto - Enfermagem, 21(1), 194-199. https://doi.org/10.1590/S0104-07 072012000100022

Cherubini, Z. A., Bosa, C. A., \& Bandeira, D. R. (2008). Estresse e autoconceito em pais e mães de crianças com a síndrome do X-Frágil. Psicologia: Reflexão e Crítica, 21(3), 409-417. https://doi.org/10.1590/ S0102-79722008000300009

Conselho Nacional de Saúde (Brasil). (2012). Resolução $n^{\circ} 466$, de 12 de dezembro de 2012. Recuperado em janeiro, 15, 2013, de http://conselho.saude.gov.br/ resolucoes/2012/Reso466.pdf

Cravinho, C. R. M., \& Cunha, A. C. B. (2015). Enfrentamento da morte fetal pela enfermagem na Abordagem Disposicional e na Teoria Motivacional do Coping. Estudos de Psicologia (Campinas), 32(2), 307-317. https://doi.org/10.1590/0103-166X 2015000200014

Fiamenghi Jr., G. A., \& Messa, A. A. (2007). Pais, filhos e deficiência: estudos sobre as relações familiares. Psicologia Ciência e Profissão, 27(2), 236-245. http://
Gohel, M., Mukherjee, S., \& Choudhary, S. K. (2011). Psychosocial impact on the parents of mentally retardation children. Anand District. Healthline, 2(2). Retrived May 10, 2015, from http://iapsmgc.org/ OA15V2I2.pdf

Gonçalves, L. C. S., Reis, L. B., \& Vicente, S. R. C. R. M. (2015). Estudo sobre Coping com mães de bebês com cardiopatia congênita: um estudo descritivo. In A. S. Nascimento \& F. H. F. Miranda (Orgs.), Da Psicologia Social à Psicologia do Desenvolvimento: pesquisas e temáticas do século XXI, (Vol.1). Jundiaí: Paco.

Grassi-Oliveira, R., Daruy Filho, L., \& Brietzke, E. (2008). Coping como função executiva. Psico, 39(3), 275-281. Recuperado em maio 10, 2015, de http://revistas eletronicas.pucrs.br/ojs/index.php/revistapsico/article/ view/3852/3368

Hassall, R., Rose, J., \& McDonald, J. (2005). Parenting stress in mothers of children with an intellectual disability: The effects of parental cognitions in relation to child characteristics and family support. Journal of Intellectual Disability Research, 49(6), 405-418. https:// doi.org/10.1111/j.1365-2788.2005.00673.x

Heiman, T. (2002). Parents of children with disabilities: Resilience, coping, and future expectations. Journal of Developmental and Physical Disabilities, 14(2), 159171. https://doi.org/doi 10.1023/A:10152195146211

Hostert, P. C. C. P., Motta, A. B., \& Enumo, S. R. F. (2015). Coping da hospitalização em crianças com câncer: a importância da classe hospitalar. Estudos de Psicologia (Campinas), 32(4), 627-639. https://doi. org/10.1590/0103-166X2015000400006

Karasavvidis, S., Avgerinou, C., Lianou, E., Priftis, D., Lianou, A., \& Siamaga, E. (2011). Mental retardation and parenting stress. International Journal of Caring Sciences, 4(1). Retrived February, 15, 2015, from http://www.internationaljournalofcaringsciences.org/ docs/Nol4_Issue1_04_Karasavvidis.pdf

Lazarus, R. S. (1993). Coping theory and research: Past, present, and future. Psychosomatic Medicine, 55(3), 234-247.

Lees, D. C. (2007). An empirical investigation of the motivational theory of coping in middle to late childhood (Unpublished doctoral thesis). Griffith University, Brisbane.

Lidz, C. S. (2003). Early childhood assessment. New Jersey: John Wile \& Sons.

Matsukura, T. S., Marturano, E. M., Oishi, J., \& Borasche, G. (2007). Estresse e suporte social em mães de crianças com necessidades especiais. Revista Brasileira de Educação Especial, 13(3), 415-428. https://doi. org/10.1590/S1413-65382007000300008

Motta. A. B., \& Enumo, S. R. F (2010). Intervenção psicológica lúdica para o enfrentamento da hospitalização em crianças com câncer. Psicologia: Teoria e Pesquisa, 26(3), 445-454. 
Motta, A. B. et al. (2015). Comportamentos de coping no contexto da hospitalização infantil. Estudos de Psicologia (Campinas), 32(2), 331-341. https://dx.doi. org/10.1590/0103-166X2015000200016

Piccinini, C. A., Frizzo, G. B., Brys, I., \& Lopes, R. C. S. (2011). Parentalidade no contexto da depressão pósparto. In C. A. Piccinini \& P. Alvarenga. Maternidade e paternidade: a parentalidade em diferentes contextos. São Paulo: Casa do Psicólogo.

Ramos, F. P., Enumo, S. R. F., \& Paula, K. M. P (2015). Teoria motivacional do Coping: uma proposta desenvolvimentista de análise do enfrentamento do estresse. Estudos de Psicologia (Campinas), 32(2), 269-279. https://doi.org/10.1590/0103-166X2015 000200011

Razurel, C., Kaiser, B., Sellenet, C., \& Epiney, M. (2013). Relation between perceived stress, social support, and coping strategies and maternal well-being: A review of the literature. Women and Health, 53(1), 74-99. https://doi.org/10.1080/03630242.2012.732681

Ribeiro, M. F. M., Porto, C. C., \& Vandenberghe, L. (2013). Estresse parental em famílias de crianças com paralisia cerebral: revisão integrativa. Ciência and Saúde Coletiva, 18(6), 1705-1715. https://doi.org/10.1590/ S1413-81232013000600022

Rooke, M. I., \& Pereira-Silva, N. L. (2016). Indicativos de resiliência familiar em famílias de crianças com síndrome de Down. Estudos de Psicologia (Campinas), 33(1), 117-126. https://doi.org/10.1590/1982027520160001000012

Seidl, E. M. F., Tróccoli, B. T., \& Zannon, C. M. L. C. (2001). Análise fatorial de uma medida de estratégias de Enfrentamento. Psicologia: Teoria e Pesquisa, 17(3), 225-234. https://doi.org/10.1590/S010237722001000300004

Silva, N. L. P., \& Dessen, M. A. (2004). O que significa ter uma criança com deficiência mental na família? Educar em Revista, (23), 161-183.

Silva, N. L. P., \& Dessen, M. A. (2003). Crianças com Síndrome de Down e suas interações familiares. Psicologia: Reflexão e Crítica, 16(3), 503-514. https:// doi.org/10.1590/S0102-79722003000300009

Skinner, E. A. (1999). Action regulation, coping, and development. In J. Brandtstandter \& R. M. Lerner (Eds.). Action and self-development: Theory and research through the lifespan (pp. 465-503). Thousand Oaks, CA: Sage.

Skinner, E. A. (2007). Coping assessment. In S. Ayers, A. Baum, C. McManaus, S. Newman, K. Wallston, \& J. Weinman (Eds.), Cambridge handbook of psychology, health and medicine (2nd ed., pp. 245-250). Cambridge, UK: Cambridge University Press.

Skinner, E. A., \& Edge, K. (2002). Parenting, motivation, and the development of coping. In L. J. Crockett (Ed.).
The Nebraska Symposium on Motivation: Motivation, agency, and the life course (pp. 77-143). Lincoln, NB: University of Nebraska Press.

Skinner, E. A., Edge, K., Altaman, J., \& Sherwood, H. (2003). Searching for the structure of coping: A review and critique of category systems for classifying ways of coping. Pychological Bulletin, 129(2), 216-269. https:// doi.org/10.1037/0033-2909.129.2.216

Skinner, E. A., \& Wellborn, J. G. (1994). Coping during childhood and adolescence: A motivational perspective. In D. L. Feartherman, R. M. Lerner, \& M. Perlumutter (Eds.), Life-span development and behavior (Vol. 12, pp. 91-133). Hillsdale, NJ: Lawrence Erlbaum Associates.

Skinner, E. A., \& Zimmer-Gembeck, M. J. (2009). Challenges to developmental study of coping. New Directions for Child and Adolescent Development, 124, 5-17. https://doi.org/10.1002/cd.239

Summers, J.A., Behr, S.K., \& Turnbull, A.P. (1988). Positive adaptation and coping strengths of families who have children with disabilities. In G.H.S. Singer \& L.K. Irvin (Eds.), Support for caregiving families: Enabling positive adaptation to disability (pp. 27-40). Baltimore: Brookes. Retrived June 4, 2015, from https:// kuscholarworks.ku.edu/bitstream/handle/1808/6253/ PPD2_Positive_Adaptation_and_Coping_8_07.pdf; sequence $=1$

Taanila, A., Syrjälä, L., Kokkonen, J., \& Järvelin, M. R. (2002). Coping of parents with physically and/or intellectually disabled children. Child: Care, Health and Development, 28(1), 73-86. https//:doi.org/10.1046/ j.1365-2214.2002.00244.x

Trute, T., Benzies, K. M., \& Worthington, C. (2012). Mother positivity and family adjustment in households with children with a serious disability. Journal of Child and Family Studies, 21(3), 411-417. https//:doi. org/10.1007/s10826-011-9492-x

Weiss, J. A., Sullivan, A., \& Diamond, T. (2003). Parent stress and adaptive functioning of individuals with developmental disabilities. Journal on Developmental Disabilities, 10(1). https//:doi.org/10.1007/s10826013-9875-2

Zanon, C., Borsa, J. C., Bandeira, D. R., \& Hutz, C. S. (2012). Relações entre pensamento ruminativo e facetas do neuroticismo. Estudos de Psicologia (Campinas), 29(2), 173-181. https://doi.org/10.1590/ S0103-166X2012000200003

Zimmer-Gembeck, M. J., \& Skinner, E. A. (2011). The development of coping across childhood and adolescence: An integrative review and critique of research. International Journal of Behavioral Development, 35(1), 1-17. https://doi.org/10.1177/01 65025410384923 
Zimmer-Gembeck, M. J., \& Skinner, E. A. (2015). Adolescent vulnerability and the distress of rejection: Associations of adjustment problems and gender with control, emotions, and coping. Journal of Adolescence, 45, 149-159. https://doi.org/10.1016/j. adolescence.2015.09.004

Zimmer-Gembeck, M. J., \& Skinner, E. A. (2016). The development of coping: Implications for psychopathology and resilience. In D. Cicchetti (Ed.), Developmental psychopathology. New York: Wiley. Retrived July 10, 2016, from https://www.pdx.edu/psy/ ellen-skinner-phd-professor-of-human-developmentdepartment-of-psychology-3

Received: May 20, 2016

Final version: December 14, 2016

Approved: February 16, 2017 